\title{
Avaliação da comunicação expressiva em crianças dos 6 aos 41 meses de idade na região norte de Portugal: Um estudo exploratório com o Early \\ Communication Indicator.
}

\author{
Assessment of expressive communication in children aged 6 to 41 months in \\ Northern Portugal: An exploratory study with the Early Communication \\ Indicator
}

Maria Cristina Antunes, Anabela Cruz-Santos

Instituto da Educação /CIEd - Universidade do Minho

\begin{abstract}
Resumo
O Early Communication Indicator (ECI) é um instrumento de medida que visa identificar, intervir e monitorizar precocemente problemas na competência comunicativa de crianças dos 6 aos 36 meses de idade. A finalidade deste estudo tem por base a tradução e adaptação do Early Communication Indicator (ECI) para o Português Europeu, administrado a 40 crianças e a análise dos resultados obtidos. Verificamos que: a) existem diferenças estatisticamente significativas nos resultados brutos totais da comunicação e nas categorias das palavras e frases em relação a todas as faixas etárias analisadas; b) a existência de um padrão de crescimento nas categorias da comunicação expressiva ao longo do desenvolvimento da criança; e c) a existência de uma evolução na produção da comunicação global. Estes resultados obtidos no ECIVersão Portuguesa são similares ao ECI original.

Palavras chave: Comunicação Expressiva; Avaliação; Early Communication Indicator - ECI, Estudo quantitativo.
\end{abstract}

\footnotetext{
Abstract

The Early Communication Indicator (ECI) is a measuring instrument that aims to identify, intervene and monitor problems in communicative competences in childrens aged 6 to 36 months. The purpose of this study is the translation and adaptation of the Early Communication Indicator (ECI) for European Portuguese, applied to 40 children and the analysis of the results it was found. We verified: a) the existence of a statistically significant differences in total results of communication and in the categories of words and multiple words for all age groups analyzed; b) the existence of a pattern of growth in the categories of expressive communication over the development of the child; and c) the existence of an increase in the production of global communication. These results obtained in the ECI-Portuguese Version are similar to the original ECI. Keywords: Expressive Communication; Evaluation; Early Communication Indicator - ECI; Quantitative study.
}

\begin{abstract}
Introdução
O estudo realizou-se com o objetivo de compreender o desenvolvimento da comunicação expressiva no que concerne aos gestos, às vocalizações, às primeiras palavras e às primeiras frases de crianças dos 6 aos 36 meses e prende-se com a constante procura de respostas profissionais relativamente ao nível de desenvolvimento da linguagem da criança em idade precoce e as constantes dúvidas surgidas ao longo de processo de desenvolvimento profissional sobre se as capacidades linguísticas da criança estão a desenvolver-se de forma equilibrada. No âmbito da pesquisa que foi efetuada nas bases de dados internacionais, o portal IGDIS apresenta o instrumento de avaliação e monitorização das competências comunicativas da criança em idade precoce: o Early Communication Indicator (ECI) (Luze, et al., 2001) que demostra capacidades de avaliação e de monitorização do comportamento comunicativo da criança de forma a delinear as estratégias de intervenção adequadas para promover o desenvolvimento da comunicação da criança e por arrasto da linguagem.

A avaliação do Early Communication Indicator (ECI) (Luze, et al., 2001) é desenhado para medir a expressão comunicativa das crianças em idade precoce. Os elementos específicos de medida da comunicação são os gestos, as vocalizações, as palavras e as frases, comportamentos exibidos durante a brincadeira com a quinta ou a casa da Fisher Price, fornecendo informação sobre o número de comportamentos comunicativos da criança.
\end{abstract}

\section{Metodologia}

Esta investigação é um estudo exploratório uma vez que os objetivos visam a análise dos processos e resultados obtidos na aplicação do instrumento de avaliação da comunicação expressiva de crianças dos 6 aos 41 meses, o ECI, desenvolvido e validado na América do Norte por Luze e al., (2001; Greenwood et 
al., 2006; Greenwood et al., 2010; Greenwood et al., 2014).

\section{Questões de investigação}

Com base na investigação realizada definiram-se as hipóteses do presente estudo:

H1: Existem diferenças significativas no desempenho comunicativo expressivo com o Early Communication Indicator - Versão Portuguesa (ECI-VP) entre as crianças quanto à idade (6 aos 41 meses);

H0: Não existem diferenças significativas no desempenho comunicativo expressivo com o Early Communication Indicator - Versão Portuguesa (ECIVP) entre as crianças quanto à idade (6 aos 41 meses);

$\mathrm{H} 2$ : Existem diferenças significativas no desempenho comunicativo expressivo com o Early Communication Indicator - Versão Portuguesa (ECI-VP) entre as crianças quanto ao género;

H0: Não existem diferenças significativas no desempenho comunicativo expressivo com o Early Communication Indicator - Versão Portuguesa (ECIVP) entre as crianças quanto ao género.

\section{Amostra}

A amostra consiste em 40 crianças da faixa etária dos 6 aos 41 meses do norte de Portugal: 3 crianças com NEE e 37 crianças sem NEE das quais 14 são do género feminino e 26 de género masculino.

\section{Instrumento de recolha de dados}

O Individual Growth and Development Indicator Early Communication Indicator-ECI (Luze et al., 2001) é um instrumento de medida usado para avaliar e monitorizar o crescimento da expressão comunicativa: gestos, vocalizações, primeiras palavras e frases, que em conjunto compõem a comunicação pré-linguística e linguística de crianças dos 6 aos 36 meses de idade, podendo alargar-se até aos 42 meses (Greenwood et al., 2006 citado em Greenwood et al., 2013; Luze et al., 2001), defendidas pelos autores após extensa análise bibliográfica (Luze et al., 2001; Greenwood et al., 2006 citado em Greenwood, et al., 2013). O desenvolvimento deste instrumento de medida da expressão comunicativa foi guiado pela seguinte diretiva: "the child uses gestures, sounds, words, or sentences to convey wants and needs or to express meaning to others", identificada por Priest e al., (1998) num estudo de vistoria a pais e profissionais, emergindo estes comportamentos expressivos num global de 15 comportamentos esperados. Assim iniciaram esta demanda a partir do Estudo 1 que tinha como objetivo: a) identificar na literatura os elementos chave da expressão comunicativa de crianças dos 0 aos 36 meses; b) desenvolver um sistema de observação destes elementos chave; c) desenvolver e comparar dois métodos alternativos que evoquem a comunicação da criança; d) e, avaliar a capacidade dos dois métodos obterem o total de comunicação da criança num curto espaço de tempo (p.385 citado em Luze et al,, 2001). O resultado do Estudo 1 foi um sistema de gravação (câmara de vídeo) dos elementos chave da comunicação (gestos, vocalizações, palavras e frases), dois promissores instrumentos de medida (Fisher Price casa e Fisher Price quinta), que alternadamente e de diferentes formas medem a expressão comunicativa; e um padrão alternativo de avaliação de situações: Naturalistic Observation Situatios (NOS), ou seja a observação da interação entre a criança, o adulto e dois brinquedos: Fisher Price casa e Fisher Price quinta.

A administração do ECI-Versão Portuguesa envolve a gestão de vários recursos, materiais e humanos. Os recursos humanos são um cuidador adulto que poderá interagir como parceiro de jogo com a criança e um outro adulto que regista os comportamentos comunicativos da criança na ficha de registo (na falta de outro adulto a gravação através da câmara de vídeo é essencial e posterior visualização e registo). Os recursos materiais são:

a) Quinta ou a casa da Fisher-Price (Figura 1), e respetivos bonecos aplicados de forma alternada, ou seja, na primeira avaliação a casa, na segunda a quinta, na terceira a casa e assim sucessivamente (Greenwood et al., 2013), são formas alternativas equivalentes para evocar o comportamento comunicativo durante o jogo (Luze, et al., 2001);

b) A sessão de avaliação ECI-VP tem lugar num cenário conveniente com poucas distrações presentes em casa ou na creche, ou seja, um espaço livre de outras crianças, outros brinquedos, sons e barulhos que possam alterar a dinâmica interativa do jogo;

c) Relógio digital ou outro dispositivo (telemóvel) possibilitador de cronometrar a sessão de avaliação;

d) Câmara de vídeo para gravar os comportamentos comunicativos da criança;

e) Lápis ou caneta para marcar/pontuar as competências da comunicação;

f) Ficha de registo $E C I$-VP;

g) Formulário da lista de verificação da administração do ECI -VP, a usar nas primeiras administrações para assegurar que são cumpridas todas as regulamentações.

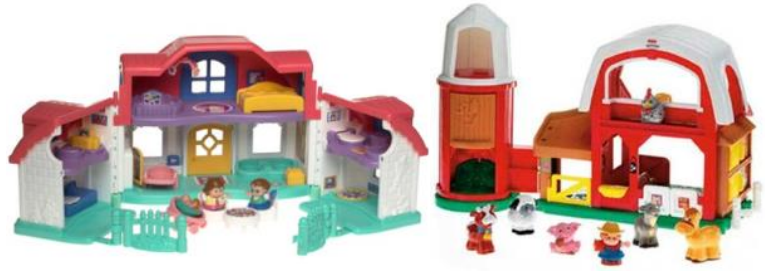

Figura 1. Casa e quinta: material do ECI-VP.

O ECI-VP é administrado numa das seguintes situações:

1. O intervencionista serve de parceiro de jogo e grava em vídeo a avaliação para mais tarde pontuar;

2. Um outro intervencionista pontua a avaliação ao vivo enquanto o outro serve como parceiro de jogo, ou;

3. O cuidador serve de parceiro de jogo enquanto o intervencionista pontua ao vivo.

O intervencionista marca o tempo da sessão para 6 minutos usando um relógio digital. Inicialmente se o intervencionista não é familiar da criança, e no caso de uma inicial sessão de avaliação, este deve interagir com a criança em variados jogos antes da avaliação com o ECI-VP para que se torne familiar. A familiaridade é 
alcançada quando a criança está disposta a interagir no jogo com o adulto (Greenwood, et al., 2013). O papel do parceiro de jogo durante a sessão do ECI-VP é encorajar a comunicação da criança seguindo a sua liderança e comentando as ações e palavras da criança. Porque o objetivo é capturar a performance comunicativa típica o intervencionista não deve dirigir ou tomar a liderança da criança, mas antes suportar os seus comportamentos comunicativos através do encorajamento e interesse.

Quanto à cotação das categorias da comunicação expressiva os gestos são cotados quando ocorre um qualquer movimento com intenção comunicativa; as vocalizações podem ocorrer isoladas ou com gestos e podem ser balbucios, arrulhos, "ah", "da", sons de animais, etc., e são cotadas quando são intencionalmente comunicativas; as palavras são sons ou sinais (linguagem gestual) produzidos pela criança que são reconhecidas e compreendidas pela pessoa que as ouve e com a intenção de comunicar algo do seu interesse; e as frases são duas ou mais palavras diferentes sonorizadas ou gesticuladas (linguagem gestual) pela criança compreendidas pelo codificador, para contar como frase as palavras/sinais devem caber juntos de forma significativa para ser considerada discurso ou sentença e serem compreendidas (Walker \& Carta, 2010). Para formar um indicador do total de comunicações sensível ao aumento da proficiência na comunicação foram usadas escalas de contagem diferentes: os gestos isolados são contabilizados com valor 1 , as vocalizações com valor 1 , cada palavra proferida pela criança com intenção de comunicar é contabilizada com valor 2 , ou seja uma palavra multiplicada por dois $(1 \times 2)$, e as frases são contabilizadas com valor 3 , ou seja uma frase multiplicada por três (1x3) (Luze, et al., 2001). Os gestos são cotados sempre que apareçam com intenção comunicativa, ou seja se aparece junto com a vocalização cotamos um gesto e uma vocalização, se aparece junto com a palavra cotamos um gesto e uma palavra, se aparece junto com a frase cotamos um gesto e uma frase. Pelo contrário quando aparece uma vocalização e uma palavra cotamos só a palavra, uma vocalização e a frase cotamos só a frase. A frequência da ocorrência de cada competência chave é pontuada a lápis na ficha de registo ao longo dos 6 minutos de avaliação. A frequência do comportamento é somada e subsequentemente dividida por 6 minutos para obter o número de respostas comunicativas por minuto.

A intervenção precoce na comunicação linguística deve considerar como as experiências recetivas e expressivas podem ser incorporadas nas estratégias de intervenção durante o início do período de desenvolvimento (Romski \& Sevcik, 2005), pois como salienta Warren, e al., (2008) o modelo transacional de desenvolvimento da comunicação e linguagem segue duas premissas básicas ao nível da intervenção: a primeira é que a quantidade e qualidade dos inputs linguístico que a criança recebe é crucial para o desenvolvimento equilibrado; a segunda é que a linha de intervenção mais eficiente depende tanto do nível de desenvolvimento em que a criança se encontra na altura da intervenção como da natureza dos objetivos a alcançar dessa mesma intervenção.

\section{Procedimento de recolha de dados}

A investigação em curso iniciou-se com o pedido de autorização aos investigadores do Juniper Gardens Children's Project para estudar o instrumento de avaliação da comunicação expressiva ECI junto da população portuguesa, e a resposta obtida foi a necessidade de realização de formação e certificação para poder aplicar e cotar o comportamento comunicativo da criança. Este processo desenvolveu-se com o estudo dos documentos disponíveis na sua página de internet e a análise de dois vídeos de aplicação do $E C I$ a uma criança que brinca com a casa ou a quinta e a técnica durante os 6 minutos de sessão. Após a visualização dos vídeos e respetiva cotação de cada categoria observada começa o processo de introdução dos dados e para isso foi criada uma plataforma online com nome da investigadora e da coordenadora da investigação. Estes resultados são um teste de avaliação da capacidade de o técnico cotar o número de comunicações por categorias com intenção comunicativa, e para conseguir a certificação ECI o investigador tem que obter valores superior a $85 \%$ e assim validar a confiabilidade de cotação da comunicação expressiva (Greenwood et al., 2011; Missall et al., 2008). Os resultados obtidos após a visualização, cotação dos comportamentos comunicativos e inserção dos mesmos na base de dados foi de $97 \%$ de confiabilidade, a investigadora e a orientadora da investigação, entre estes e os resultados obtidos pelos autores do instrumento.

A escolha do local de aplicação do instrumento de avaliação da competência comunicativa $E C I$-VP deve-se à proximidade social com a amostra. Finalizado o processo de consentimentos para recolha de dados procedeu-se ao levantamento de dados relativamente às datas de nascimento e presença, ou não, de necessidades educativas especiais, e às habilitações literárias dos pais. A maioria das avaliações foi entre a investigadora e a criança e outras preferiram a brincadeira com o cuidador mais próximo: a educadora, a auxiliar de ação educativa, a avó, a tia e/ou a irmã. Foram realizadas 3 avaliações com o ECI-VP alternadas entre a casa, a quinta e por último a casa, ao longo de 6 meses com intervalo de 2 meses, ou seja, em média cada criança foi avaliada de 2 em 2 meses. Após finalizadas as avaliações regista-se a quantidade de respostas comunicativas por categoria ao longo dos 6 minutos na ficha de registo ECI-VP, traduzida e adaptada do ECI Data Sheet original, e a subsequente soma do total de cada categoria (gestos, vocalizações, palavras e frases) a dividir pelos seis minutos de aplicação do instrumento $E C I$-VP e obtêm-se os resultados globais de comunicações por minuto.

\section{Análise dos resultados.}

A proposta desta investigação é examinar a validade da avaliação e monitorização da comunicação expressiva de crianças em idade precoce com o ECI-VP. A análise de dados segue a linha da idade cronológica das crianças aquando das avaliações, ou seja, a primeira avaliação foi realizada em meados de março de 2015 tendo a criança mais nova 6 meses e a mais velha 37 meses, as seguintes avaliações ocorreram em média de dois em dois meses. 
A tabela 1 revela os resultados brutos de comunicações cotadas: em relação aos valores mais baixos é de 3,5 comunicações por minuto na primeira avaliação, 4 comunicações por minuto na segunda e 4,5 comunicações por minuto na terceira e referem-se à criança mais nova de 6 meses, com o valor cotado obtido a aumentar da primeira à terceira avaliação com o ECIVP. Quanto ao valor máximo cotado verifica-se uma proximidade de resultados, com 45,2 comunicações por minuto na primeira avaliação, 43,2 na segunda e 45,7 na terceira.

Tabela 1. Análise dos resultados brutos obtidos no ECI-VP.

\begin{tabular}{|l|c|c|c|c|c|}
\hline Resultados brutos obtidos no $E C I$-VP & Média & $\begin{array}{c}\text { Desvio } \\
\text { Padrão }\end{array}$ & Mín. & Máx. & N \\
\hline ECI-VP casa 1 & 16,28 & 10,75 & 3,5 & 45,2 & 40 \\
\hline ECI-VP quinta 2 & 20,19 & 10,83 & 4,0 & 43,2 & 40 \\
\hline ECI-VP casa 3 & 22,08 & 11,43 & 4,5 & 45,7 & 40 \\
\hline
\end{tabular}

Os resultados obtidos em cada categoria da comunicação expressiva obtida com os estudos levados a cabo por Luze, e al. (2001), Greenwood e al., (2006 citado em Greenwood et al 2013), Buzhardt, e al. (2010) e Greenwood, e al. (2013) representado na figura 2 antecede o nosso gráfico de desenvolvimento e crescimento da comunicação expressiva das categorias gestos, vocalizações, palavras e frases com o gráfico da figura 3 , a partir dos resultados brutos obtidos com os 3 momentos de avaliação das 40 crianças da nossa amostra com o ECI-VP, com o objetivo de demostrar que existem padrões de consistência entre os resultados obtidos com o ECI original e ECI-VP no que respeita aos padrões de crescimento por categoria.

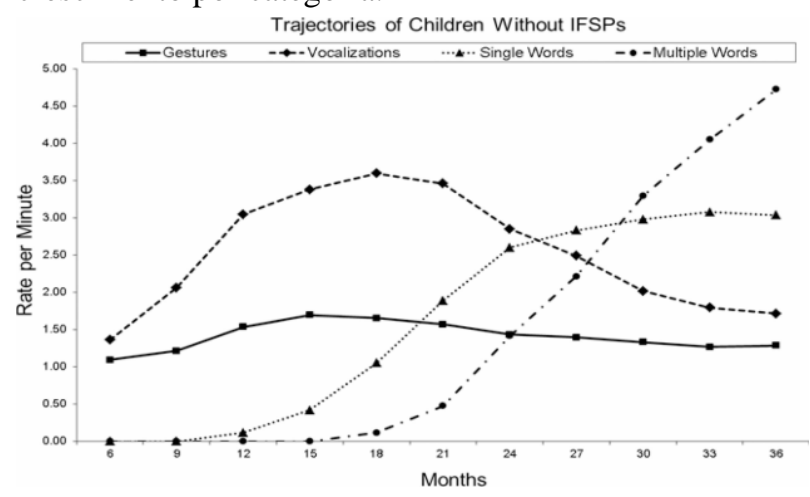

Figura 2. Trajetória do crescimento da comunicação expressiva com o ECI original por categorias (Greenwood et al., 2011).

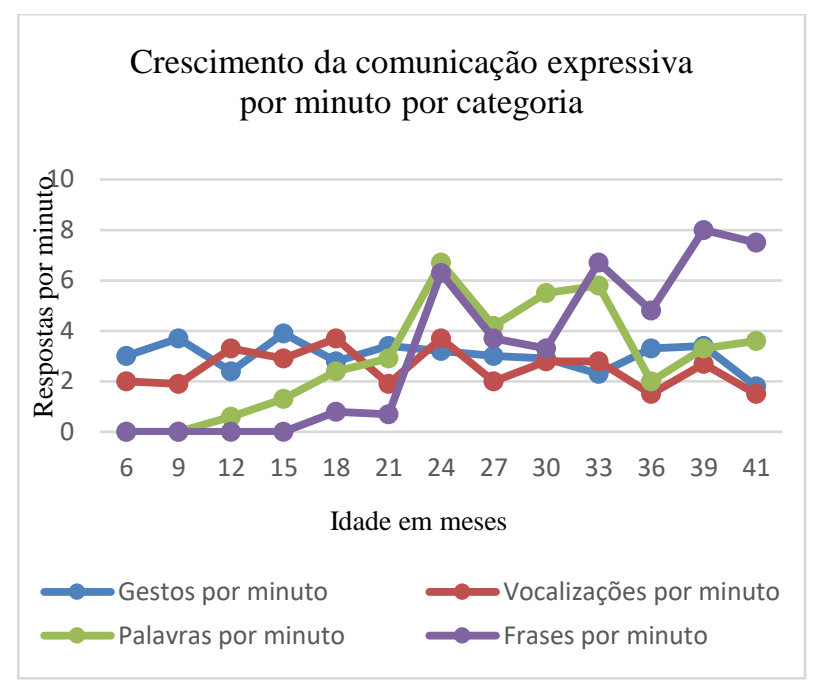

Figura 3. Trajetória de crescimento da comunicação expressiva com o ECI-VP por categorias.

Da análise dos dois gráficos verificamos que os gestos do $E C I$ original obtém valores brutos médios entre 1 e 2 por minuto ao longo de todo o período de crescimento tal como os gestos no ECI-VP embora os valores sejam entre 2 e 4 gestos por minuto; o mesmo acontece com as vocalizações em relação à presença desta categoria comunicativa ao longo de todo o período de desenvolvimento da comunicação e linguagem, e com o $E C I$ original as vocalizações estão entre 1,5 e 4 por minuto assim como o ECI-VP e existe um pico nesta categoria entre os 15 e os 21 meses em ambos os ECI's; no que concerne às palavras verifica-se o início aos 12 meses em ambos os ECI's e aumenta ao longo do tempo até estagnar nas 3 comunicações por minuto a partir dos 27 meses; quanto às primeiras frases verifica-se que em ambos os ECI's as crianças iniciam a primeira frase com duas ou mais palavras por volta dos 18 meses e aumenta ao longo do tempo até às 5 frases por minuto em média. Verificou-se a existência de diferenças significativas no desempenho comunicativo expressivo entre as crianças quanto à faixa etária dos 6 aos 41 meses de idade uma vez que há uma trajetória de evolução contínua no uso das categorias para comunicar, ou seja existe uma ordem temporal crescente nesta aquisição (gestos e vocalizações até aos 12 meses, gestos, vocalizações e palavras até aos 18 meses, e gestos, vocalizações, palavras e frases dos 18 meses em diante, em média). Como constatamos, o uso das categorias da comunicação sofrem aumentos e diminuições ao longo do desenvolvimento da criança: a forma de comunicar consiste na produção por minuto em média entre 2 a 4 gestos por minuto desde os 6 aos 18 meses, em média 3 gestos entre os 18 e os 27 meses, e entre 2 a 3 gestos por minuto entre os 27 e os 41 meses; a forma de comunicar consiste em média entre 2 a 4 vocalizações por minuto desde os 6 aos 18 meses, em média entre 4 e 2 vocalizações por minuto entre os 18 e os 27 meses, e entre 2 a 3 vocalizações por minuto entre os 27 e os 41 meses; a forma de comunicar em média entre 0 e 2 palavras por minuto desde os 6 aos 18 meses, em média entre 2 e 5 palavras por minuto entre os 18 e os 27 meses, e entre 4 palavras por minuto entre os 27 e os 41 meses; e a forma de comunicar em média é 0 frases 
por minuto desde os 6 aos 18 meses, em média entre 1 e 4 frases por minuto entre os 18 e os 27 meses, e entre 4 e 7 frases por minuto entre os 27 e os 41 meses.

Após a análise dos resultados brutos da comunicação expressiva por categorias com o ECI-VP e após verificar a existência de diferenças significativas no desempenho comunicativo expressivo com o ECI-VP entre as crianças quanto à faixa etária dos 6 aos 36 meses de idade verificou-se que há uma evolução no desempenho da comunicação expressiva, hipótese já demostrada pelos autores do instrumento em questão como se verifica com a figura 4 que é nada mais do que os resultados brutos da comunicação expressiva da avaliação com o ECI original de 5883 crianças em 26 programas de intervenção precoce de 2 estados americanos.

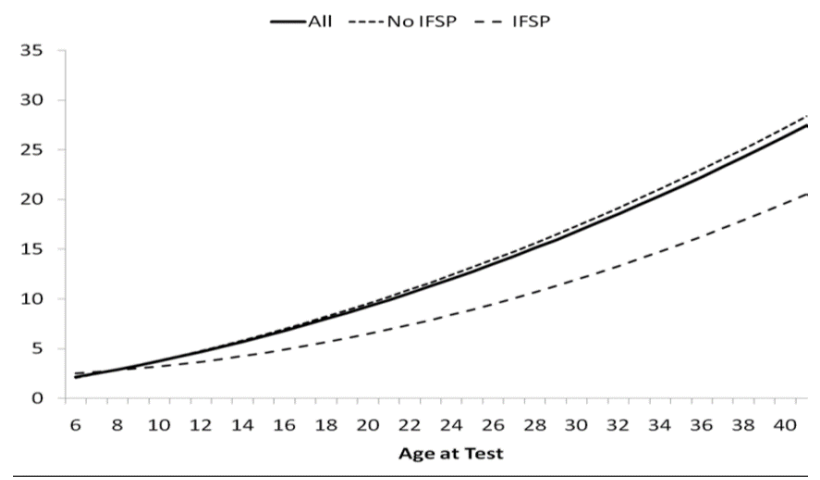

Figura 4. Trajetória do total de comunicações por minuto com o ECI original (Greenwood, Carta \& McConnell, 2011).

Os resultados brutos de comunicações cotados com o ECI-VP representados na figura 5 demostram o crescimento curvilíneo da comunicação expressiva das 40 crianças do norte de Portugal avaliadas ao longo de 3 sessões com intervalo de 2 meses. Concluímos, portanto, que existem diferenças significativas no crescimento da comunicação expressiva entre as crianças da faixa dos 6 aos 41 meses de idade.

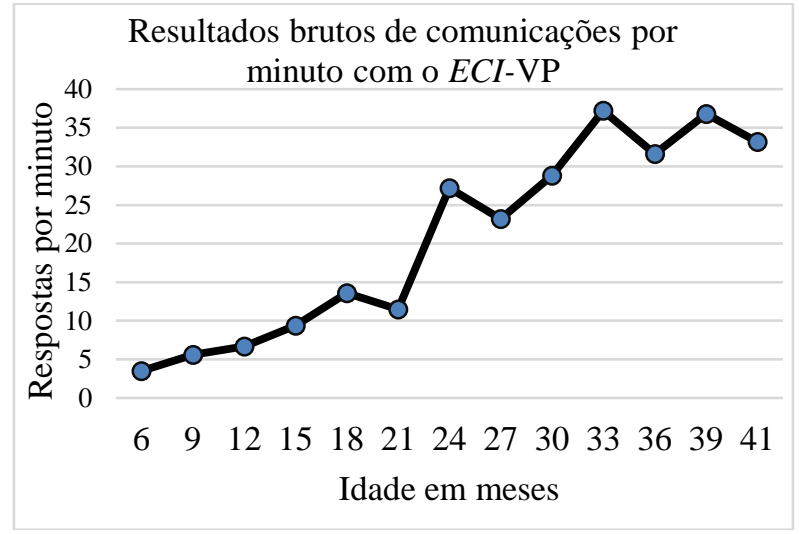

Figura 5. Trajetória dos resultados brutos de comunicações por minuto com o ECI-VP.

Destes resultados na proficiência nas categorias da comunicação resulta a trajetória de crescimento da comunicação expressiva global, ou seja, dos 6 aos 12 entre 3 a 7 comunicações por minuto; entre os 12 e os 24 meses entre 7 e 27 comunicações por minuto; dos 24 aos 41 meses de 27 a 32 comunicações por minuto.

Verificou-se que quanto ao género as crianças obterão médias de resultado global de comunicações por minuto para o género feminino de 19,56 e para o género masculino de 19,50, demostrando que não existem diferenças significativas no desempenho comunicativo com o ECI-VP, assim como nos resultados de investigação reportados em Geenwood e al., (2010), ou seja, esta não é uma variável preponderante no estudo.

Quanto à fiabilidade de obtenção de resultados foram analisados independentemente 30 vídeos das três fases de avaliações ECI-VP, 10 sessões de avaliação por cada fase, por um segundo investigador ou seja $36 \%$ do total da amostra. A média de concordância entre investigadores é auferida a partir da utilização da segunda tabela da ficha de registo ECI-VP, e os resultados foram $83,5 \%$ de concordância entre investigadores quanto ao total de comunicações por minuto. Valores similares para as categorias da comunicação foram encontrados, nomeadamente $81,8 \%$ para os gestos, $82,7 \%$ para as vocalizações, $83,3 \%$ para as palavras e $88,1 \%$ para as frases.

\section{Conclusões}

Concluímos que aos 6 meses de idade a criança usa gestos e vocalizações para comunicar; que por volta dos 12 meses de idade começa a usar as palavras e mantém os gestos e vocalizações; e que a partir dos 18 meses de idade a criança começa a introduzir no seu diálogo expressivo as primeiras frases em consonância com os gestos, vocalizações e palavras, o que revela padrões de consistência entre os resultados obtidos com o ECI original e o ECI-VP como referimos na análise inferencial, embora obtenhamos valores mais elevados nos resultados brutos por categorias e, consequentemente, nos resultados brutos globais. Demonstra-se também que à medida que a criança adiciona e usa as palavras e as frases diminui o número de vezes que usa os gestos e as vocalizações, mas denotase que estão presentes ao longo do desenvolvimento e crescimento da comunicação expressiva, tal como verificaram os autores do ECI (Carta, et al., 2002; Greenwood, et al., 2013; Luze, et al., 2001). Coletivamente os resultados suportam a partilha de propriedades das ferramentas chave do ECI-VP de um continuo crescimento entre os gestos, as vocalizações, as palavras e as frases.

\section{Referências bibliográficas}

Buzhardt, J., Greenwood, C., Walker, D., Carta, J., Terry, B., \& Garrett, M. (2010). A Web-Based Tool to Support Data-Based Early Intervention Decision Making. Topics in Early Childhood Special Education, 201-213.

Carta, J. J., Greenwood, C. R., Walker, D., Kaminski, R., Good, R., McConnell, S., \& McEvoy, M. (2002). Individual Growth and Development Indicators (IGDIs): Assessment that Guides Intervention for Young Children. Em M. Ostrosky, \& E. Horn, Assessment: Gathering meaningful information. TheYoung 
Exceptional Children Monograph Series (pp. 1-20). Longmont: Sopris West.

Greenwood, C. R., Carta, J. J., \& McConnell, S. (2011). Advances in Measurement for Universal Screening and Individual Progress Monitoring of Young Children. Journal of Early Intervention, 254-267.

Greenwood , C., \& Carta, J. (2011). Response to Intervention (RTI) with Infants/Toddlers Early Communication. Utah Conference on Effective Practices for Teachers and Human Service Professionals: Interventions Across the Lifespan. Utah State University: Logan UT.

Greenwood, C., Walker, D., Buzhardt, J., Howard, W., McCune, L., \& Anderson, R. (2013). Evidence of a Continuum in Foundational Expressive Communication Skills. Early Child Res Q., 540-554.

Luze, G., Linebarger, D., Greenwood, C., Carta, J., Walker, D., Leitschuh, C., \& Atwater, J. (2001). Developing a General Outcome Measure of Grow in the Expressive Communication of Infants and Toddlers. School Psychology Review, 383-406.

Romski , M., \& Sevcik, R. (2005). Augmentative Communication and Early Intervention. Myths and Realities. Infants \& Young Children, 174-185.

Romsky, M., Sevcik, R., \& Adamson, L. (2011). Parent Perceptions of the Language Development of Toddlers With Developmental Delays Before and After Participation in Parent-Coached Language Interventions. American Journal of Speech-Language Pathology, 111118.

Ruiz, J. R., \& Ortega, J. L. (1993). As Perturbações da Linguagem Verbal. Em R. Bautista, Necessidades Educativas Especiais (pp. 83-108). Lisboa: Dinalivro.

Warren, S., Fey, M., Finestack, L., Brady, N., BredinOja, S., \& Fleming, K. (2008). A Randomized Trial of Longitudinal Effects of Low-Intensity Responsivity Education/Prelinguistic Milieu Teaching. Journal of Speech, Language, and Hearing Research, 451-470. 